\section{ON THE UNITY OF THE SYPHILITIC POISON.*}

By S. MESSENGER BRADLEY, F.R.C.S.,

Lecturer on Comparative Anatomy, etc., in the Royal School of Medicine Manchester.

No fact in surgery is better established than the auto-inoculability of soft chancre. Experiments have also long since proved that the soft sore may be conveyed by means of inoculation from one individual to another. For a time, the inoculability of the hard sore was denied; but, more recently, experiments have demonstrated the fact that the hard or infecting chancre is in like manner capable of inoculation upon a virgin subject, there giving rise to a sore similar in nature to the original lesion, and in course of time, like it, followed by a train of constitutional symptoms. Furthermore, it is admitted by the so-called dualists, that a sore is occasionally grafted upon a subject already syphilised by matter taken from a true infecting chancre. In this case, they affirm that the sore so produced is invariably soft. This circumstance has not led them to infer the common origin of the two lesions, but that the soil (i.e., the system of the syphilised subject) is so changed as altogether to modify the character of the inoculated virus without converting it into the "materies" of true soft chancres. It is manifest that, in order to prove the common origin of the syphilitic poison-in other words, to prove the unity of syphilis-it is necessary to produce a soft sore upon a virgin subject by direct inoculation from a hard sore, i.e., to produce a sore indefinitely, capable of auto-inoculation and never followed by constitutional symptoms. I have made numerous experiments to ascertain whether this was or was not possible, and here give the results of these experiments.

My subjects were monkeys, kittens, and guinea-pigs; the virus which I employed was obtained from cases of syphilis met with in private practice, in the Lock Hospital, and in the venereal wards of the Manchester and Chorlton Workhouses. I obtained the matter for inoculation by scraping the surface of the sore, prior to cicatrisation, with either a piece of glass or an ivory vaccination-point.

The great majority of my experimerits gave negative results; these I do not record, as they prove nothing. In two instances (one in a guineapig, and one in a kitten), the inoculation was followed, after the interva of two or three weeks, by local thickening at the site of puncture, and, later, by the outbreak of constitutional symptoms. The guinea-pig died within a month from the commencement of the basal thickening of the chancre, with disorganisation of one eye and extensive ulceration about the mouth and soft palate; the kitten I killed at the end of the eighth week, and found syphilitic gummata in the kidneys and liver.

Omitting failures and the two cases of syphilis mentioned above, I obtained the three following successful results.

EXPERIMENT I.-On March 28th, I871, I took some virus from a syphilitic sore upon a female, aged 19. The sore, which was situated upon the left labium, was first noticed three weeks before. It presented a well-defined base, and very scanty secretion. There were mucous patches about the anus, and a papular syphilitic eruption upon the arms and legs. A kitten was inoculated with this matter the same day, upon the inner side of the shaved haunch. On the second day, there was evident irritation at the site of inoculation, which, on the sixth, had developed into a typical soft chancre. Matter taken from this sore was inoculated upon the opposite thigh, where it produced a precisely similar result. On July 27 th, the chancres were both healed; there was no adenopathy, nor constitutional symptoms.

EXPERIMENT II. - On April 3 rd, I took virus from a syphilitic female, aged 22. The sore, situated upon the labium, had a hard base. There were multiple inguinal adenopathy, and syphilitic sore-throat. I inoculated a guinea-pig with this virus the same day, upon the ear and haunch. The ear gave a negative result. On the eighth day, the haunch presented a characteristic soft sore, which freely suppurated. I successfully inoculated the same guinea-pig in two places, with matter taken from this sore.

July 27 th. - The chancres were all healed. There was no hardness at the bases of cicatrices, and no constitutional symptoms.

EXPERIMENT III. - Virus was taken from a male, aged 26, on May 17th, 1871. The chancre was situated behind the corona glandis, and had a well-marked cartilaginous base. There was multiple inguinal adenopathy. The sore had appeared fifteen days before, and three weeks after an impure connection. At the present date (July 28th), the patient is covered with a papular syphilitic exanthem. I inoculated a guinea-pig the same day with this virus in three places; only one puncture, however, was successful. The resulting chancre was not

* Read before the Surgical Section at the Annual Meeting of the British Medical Association in Plymouth, August, 1871 . mature until the fourteenth day, when it suppurated freely. With matter taken from this sore, I succeeded in inoculating this same guineapig and a companion rodent, which chancres in their turn gave rise to inoculable matter. On July $27^{\text {th }}$, the chancres had healed. There were no constitutional symptoms.

It is necessary for me to state that, in these experiments, the initial lesion was never irritated by any application; I merely used the secretion obtainable from the surface of the untreated sore. When the sore was irritated by savine, it was much easier to procure abundant, and, as a rule, readily inoculable pus.

I never succeeded in obtaining positive results with matter taken from a phagedænic sore, or by scraping the surface of one which was entirely void of all secretion.

Such are the results which I have obtained; and, supposing my experiments to be verified by future observers, I do not see how we can refuse to admit the correlation, the common origin, and the convertibility of the two sores. Undoubtedly this convertibility is very seldom witnessed, the tendency being, here as elsewhere, to "breed true." Noting the fact that grave constitutional symptoms were nearly always preceded by a serious and obstinate character of initial lesion, and that a mild and benignant attack of secondaries was nearly always preceded by a slight and readily healed sore, Carmichael, Bassereau, and others, described no less than four perfectly well-defined classes of syphilitic sore, each followed by its own peculiar train of constitutional symptoms. The rarity with which the convertibility of which I speak is witnessed, need not surprise us. It is, indeed, not without its parallel in medical literature; for we meet with a strictly analogous phenomenon in the clinical history of the vegetable parasites. It may, indeed, be held as settled, that the fungi infesting the human body are all interchangeable ; thus it has been proved that the mycelium of aspergillus is produced by the germination of the achorion; that favus is caused by the implantation of the torula; that favus may assume the form of tinea tonsurans; that tinea tonsurans is produced from tinea circinata, and, vice versâ, that tinea versicolor is produced by the implantation of the fungus of tinea tonsurans, or from the oidium; that the aërial spores of penicillium develope into torula ; that sarcina may develope from the spores of penicillium; that sarcina, aspergillus, and penicillium are derivable from the same source; that torula may assume the form of mucor; and many more facts of the same kind, all tending to complete the chain which connects in one family group the highest with the lowest fungus. Yet it must not be supposed that because this power of convertibility exists, it often takes place in the human subject; on the contrary, it very rarely takes place ; for, says Dr. Tilbury Fox, "the upshot of the matter is this : that the achorion, even if identical in nature with other epiphytes, cannot be expected to produce upon the human surface any variety of tinea other than favus, except as the rarest phenomenon; and inasmuch as it is the most developed condition of favus in which the spores become nucleated, budding is apparent and fructification attempted; the soil which suffices for other phases will not conduce to the retrograde growth of achorion, which has the tendency (alike common to fungi) to reproduce itself"; and, further, after demonstrating the common origin of sycosis, tinea tonsurans, and favus, he adds : "there is no ground for rejecting the identity of tinea favosa and tinea tonsurans in our inability to produce artificially the one from the other, except but very rarely." It seems probable that the same causes which thus operate in this low region of the vegetable kingdom (these causes being differences in the soil and in the age, etc., of the seed) are the active agents in determining the character of the syphilitic sore.

The precise force which each of these causes exercises, we are not yet in a position to decide. There is some reason for believing that the interchange of character between the two sores is more frequently witnessed than is commonly believed. I may quote in support of this statement, the impartial evidence given by "the Committee appointed by the Secretary of State for War and Board of Admiralty to Inquire into the Pathology and Treatment of the Venereal Diseases." At page 9 of their report, it is stated that "'hard sores do not necessarily contaminate the constitution, while, on the other hand, constitutional symptoms occasionally follow the presence of a sore which might have been regarded as a simple local sore by a practised observer."

After all, I do not consider this question of unity or duality to be one of much practical value. Granted that these experiments, when verified by the experience of others, demonstrate the unity of the poison, none the less will it be true that the original virus has given rise to two diseases, which are for all practical purposes entirely dissimilar, differing in symptoms, in prognosis, and in treatment. At the same time, I cannot admit that this fact of unity is entirely a sterile and useless fact, for two reasons ; in the first place, the essential oneness of the syphilitic poison being proved, it clearly follows that we should in all cases await the appearance of constitutional symptoms (the first of which may pro- 
bably be regarded as the multiple inguinal adenopathy, always present when infection of the system is about to take place) before commencing a course of mercurial treatment; and, in the second piace, it should make us more guarded in prognosticating constitutional infection in cases of hard chancre, and certain immunity from all secondary symptoms in undoubted cases of soft sore.

\section{THE TRIALS AND DIFFICULTIES OF A HEALTH- OFFICER.*}

\section{BY DA VID DA VIES, M.R.C.S.} Inspector of Health for Bristol.

THE appointment of health-officers being of recent date, and not yet of universal adoption by the great centres of population, a few words on the difficulties and trials peculiar to such officers seem to be desirable, especially at the present time, when we are promised better legislation on the subject, which, however, from session to session is deferred -our legislators having hitherto found other matters to be of more importance than the health of the people. Without further preamble, I will enumerate those difficulties as they have cropped up in my own experience.

I. The want of Early Information of the Occurrence of Infectious Disease in a Family or Neighbourhood. - At present this information is only at the command of a health-officer through the unpaid courtesy of his professional friends, or by keeping up a continual system of espionage over other people-a system which is never pleasant, and one that often proves unsuccessful until much mischief has been done by the spread of disease.

2. The Confused State of our Sanitary Lazos, many valuable Enactments being Permissive and not Compulsory.-As an instance of the complete manner in which our laws have failed to point out the different authorities for doing certain imperative sanitary work, take the building of hospitals for the isolation of persons suffering from infectious diseases. Under the Sanitary Act of 1866 , boards of health may build such hospitals. Are these for the admission of paupers or not? or must paupers be provided for by the respective unions? Hence arise letter-writing and disputes between nuisance-authorities and boards of guardians, whilst small-pox or other fatal diseases may be spreading far and wide; each party considering its chief duty to be that of keeping down the rates. These permissive clauses have produced such bad results that I know at the present time a large union containing a very large workhouse without a single ward for the isolation of a case of infectious disease either in the workhouse or out of it ; and I knew until very lately a large and important city without any such ward.

3. The Separation betzeen the Registration of Deaths and the Sanitary Authorities. - The latter cannot get early intimation of death from the local registrars without paying such sums as the latter may please to charge for the information. I find no fault with the registrars, as with them it is a mere matter of business; but it is evident that the system is wrong, and that the deaths registered should as soon as possible be submitted to the scrutiny of the health-officer.

4. The Division of Nuisance-Authorities, and the want of a Central Power to make them act in unison. - I will here narrate a remarkable instance of this. From the county of Somerset a brook, which was within the memory of man a clear trout-stream, runs through a part of Bristol. A nuisance has been created by a glue-factory in the county, so as to render this brook a veritable Styx. It contained black mud saturated with animal matter, which in many places was a yard deep, and which, seething under the summer sun, gave out thousands of cubic feet of poisonous gases. The nuisance was made by $\mathrm{A}$ in the county. It then ran into the brook which formed the boundary between the lands of $B$ and $C$, who had no hand in creating the nuisance. It then ran through a populous district under the jurisdiction of the Bristol Board of Health. The Board of Health called upon the guardians of the poor for that part of the county to abate the nuisance. In compliance with this the guardians, after taking the best legal advice, required $\mathrm{B}$ and $\mathrm{C}$ to cleanse the brook in the county and remove the nuisance. $B$ and $C$, who were certainly innocent parties, pleaded nonliability. The county magistrates, after hearing the case argued by eminent counsel for some hours, made an order on B and C, because the nuisance was found on their land. $B$ and $C$ complied. Within the district of the Board of Health, D was in the same position as B and $\mathrm{C}$ in the county. The Board of Health summoned $\mathrm{D}$ before the Bristol magistrates, who dismissed the case, stating that $\mathrm{D}$, being an

* Read before the Public Medicine Section at the Annual Meeting of the British Medical Association in Plymouth, August 137 r innocent party, was not liable, but that the Board of Health must find out the originator of the nuisance, who lived out of the jurisdiction of the Bristol magistrates, and had certainly no right to enter the land of $\mathrm{D}$ to remove the nuisance. Thus we have one law in the county of Somerset and another in Bristol ; and the brook is now becoming as great a nuisance as ever. Which legal authority is right, is not for me to determine : either side is made to appear plausible in the hands of counsel. I could adduce other instances of want of unison from divided authority and the consequent failure of health-authorities.

5. The Non-Adoption by our Profession of an Uniform Nosology.Hence the returns of deaths (occasionally even when made by able men) would puzzle wiser heads than mine. Some of our confrères will insist on calling a bad case of enteric fever "typhus", and a mild case of typhus goes by the name of "typhoid", or either may be disguised un. der the milder name of "gastric fever". Others enter as fever on the death-returns every case which has terminated with febrile symptoms and coma, although the cause of all the symptoms may have been disease of the kidney or some other important organ. Others of our body have peculiar views of the identity of common sore-throat, scarlet fever, and diphtheria. During the year, a health-officer, if he adopt a modern and specific nosology, must be often hurt by the returns of the Registrar-General, by which he will be informed that typhus, or perhaps cholera, has visited his district, although he is morally certain that neither has done so. But enough of this; let us hope that when we have one portal for our profession these differences will disappear.

6. The importance attributed by the Public to the mere Figures of the Registrar-General, without regard to Locality, Causes of Death, or the Ages of the Dead.- One district may be celebrated as a residence for invalids on account of its bracing air. Lethal atmospheric influences, such as prevailed in the spring of 1870, may sweep over this locality, and, by capillary bronchitis, destroy the old and the feeble in large numbers. During the same year that pest of our race, which heeds no sanitary conditions at our command-scarlet fever-may, like a destroying angel, gather the finest of our blossoms. Or a locality may be so celebrated for its hospitals and the medical and surgical attendance procured there, as to attract to it such a large number of persons suffering from fatal diseases and injuries, as, in conjunction with the workhouses, to raise the deaths in public institutions to 14 or 15 per cent. of the total. The public look only at the totals of the RegistrarGeneral, and shake them in one's face, like Shylock his bond. When an adverse combination, due only to atmospheric influences, or an uncontrollable epidemic happens, the public wiil have their say about it. Newspaper editors write learned articles on the subject, which, as a rule, are very wide of the mark. Every one that can wield a pen writes to the local papers pointing out the cause of it all. Even sometimes an old-fashioned member of the medical profession will join the hue and cry, and for ever condemn all that is local. The health-officer is everywhere greeted with anxious and dubious inquiries. The public evidently think somebody ought to be hanged; and he is the most likely victim. He walks about like a "thing of shame", supported only by his mens conscia recti. He knows the lives he has saved; the public know how many they have lost. No one appreciates more highly the intellect brought to bear by the Registrar-General on the figures in his possession than myself. Had that high authority been furnished with further information regarding distant localities, and if he could by any process of elimination distinguish the deaths which are truly local, I respectfully conclude that many of his valuable quarterly reports would differ widely from the past-that some localities reputed healthy would sink to the bottom of the list, and others now reputed as unhealthy would correspondingly rise ; that many of our country districts which send their sick to die in the large towns would change places with the latter. But the management of figures where there are so many modifying factors is a herculean task.

7. The Death of many Infants under One Year Old is one of the greatest troubles of a Health. Officer in Large Towns-The majority of these deaths arise from causes over which a health-officer has no control, which are moral rather than physical in their origin. In all large towns there is the production of offspring by individuals who have no moral right to the privilege. They have not the physical means to support them, nor have they the desire to perform the duties of parents to them after they are born. They are viewed by many as unwelcome interlopers on their sensual enjoyments; and, I am afraid, that occasionally grim and undeserved poverty destroys the paternal and maternal instinct. Many die from sheer neglect and want of breast-milk or other appropriate food ; others die from exposure, without proper clothing, to our variable climate ; but a vast number die from want of medical advice and good nursing when suffering from diseases incidental to childhood, such us measles and whooping-cough. When either of these diseases sweeps over a poor locality in the cold spring 